\title{
FEMTO-PHOTONICS: OPTICAL TRANSDUCERS UTILIZING NOVEL SUB-WAVELENGTH DUAL LAYER GRATING STRUCTURES
}

\author{
Dustin W. Carr, Gregory R. Bogart, Bianca E. N. Keeler \\ Sandia National Laboratories \\ Albuquerque, NM 87185
}

\begin{abstract}
We have fabricated and measured a novel grating structure consisting of two levels that can be actuated relative to one another. Near-field optical effects result in strong sensitivity of the zero-order diffraction efficiency, making this useful as a transducer for sensing very small amounts of relative motion. We have observed a noise floor of 100 $\mathrm{fm} / \mathrm{Hz}^{1 / 2}$ at $100 \mathrm{mHz}$.
\end{abstract}

\section{INTRODUCTION}

Deformable diffractive optical structures have been studied for many years, mostly for applications relating to video displays or communications [1]. These structures have traditionally relied upon first-order gratings with feature dimensions significantly larger than the wavelength of light with which they are designed to interact. Recent studies have looked at a different type of diffractive structure that utilizes near-field optical effects that are very sensitive to the motion of the grating elements [2]. These designs result in optical resonant structures that can modulate the intensity of the reflected or transmitted zero orders, while moving only fractions of a wavelength. Such devices are very sensitive to wavelength, and could thus be used as tunable elements for spectrometry, as well as communications or inertial sensing.

Device designs utilizing multi-layer sub-wavelength gratings that can be translated in-plane have been studied computationally in the past [3]. In this work, we study the optical and mechanical properties of fabricated structures, shown schematically in Figure 1. While precise correlation with the optical theory is difficult, we have demonstrated the strong sensitivity to motion that has been predicted in the past. Of particular interest for our applications is the use of this device as a transducer for measuring very small amounts of relative motion between the two grating levels. Such a structure could be used in an inertial sensor system. Previous work has detailed the advantages of using an optical transducer for this application [4].

\section{EXPERIMENTAL DETAILS}

An SEM micrograph of a released dual grating structure is shown in Figure 2, with the bottom grating hidden beneath the top silicon layer. The grating elements of both layers are grounded to the substrate. Comb-drive fingers enable electrostatic lateral actuation of the top grating, while parallel plate electrodes on the first silicon layer apply forces vertically. Simultaneous actuation of these two sets of electrodes allows us to map out the optical response of the structures across a large operating range.

To experimentally observe the motion in the device, a $636 \mathrm{~nm}$ laser is focused on the device, and the reflected signal is detected using a balanced detector. A reference beam is also incident on the surface near the device, enabling the subtraction of noise related to the experimental apparatus.
With this setup, we are able to study the static and dynamic properties of the devices. Studying the mechanical resonant response enables us to verify the mechanical and electrical properties, which we can then use to get a precise measure of the amplitude of motion at lower frequencies. The measured resonant frequencies of these devices are typically within $1 \%$ of those predicted using finite element analysis, an indication of the excellent control we have in the precision processing of these devices.

Figure 3 contains plots of the reflected intensity as a function of displacement from the rest position for the transverse electric (TE) and transverse magnetic (TM) modes, where the transverse direction is defined as perpendicular to the grating lattice vector. While the range of motion is very slight, we see a substantial change in the intensity of the reflected signal, approaching $15 \%$ modulation, normalized to the peak reflectance. This data is obtained by applying voltages to the two electrodes, and then translating these voltages into the known deflections of the device, which are calibrated through the use of computational models, along with dynamic resonant mode data. In this data, The two gratings have a period of $720 \mathrm{~nm}$, with a silicon fill factor of approximately $2 / 3$.

Figure 4 is an amplitude spectral density plot for a device that is being driven at $10 \mathrm{~Hz}$. The total amplitude of motion is approximately $100 \mathrm{fm}$, while the noise level is 130 $\mathrm{fm} / \mathrm{Hz}^{1 / 2}$. This remarkable sensitivity at such low frequencies is largely enabled by the use of reference optical detection, which defeats many potential sources of $1 / \mathrm{f}$ noise that becomes dominant at these levels.

Sandia is a multiprogram laboratory operated by Sandia Corporation, a Lockheed Martin Company, for the United States Department of Energy's National Nuclear Security Administration under contract DE-AC04-94AL85000.

1. O. Solgaard, F. S. A. Sandejas, and D. M. Bloom, "Deformable grating optical modulator," Optics Letters, 17, 688 (1992).

2. B. E. N. Keeler, D. W. Carr, J. P. Sullivan, T. A. Friedmann, and J. R. Wendt, "Experimental demonstration of a laterally deformable optical NEMS grating transducer," Optics Letters, accepted for publication (unpublished).

3. W. Nakagawa and Y. Fainman, "Design of tunable optical cavity based on near-field optical coupling between subwavelength periodic nanostructures," 2003 IEEE/LEOS International Conference on Optical MEMS (2003) pp. 111112.

4. R. L. Waters and M. E. Aklufi, "Micromachined FabryPerot interferometer for motion detection," Applied Physics Letters, 81, 3320 (2002). 


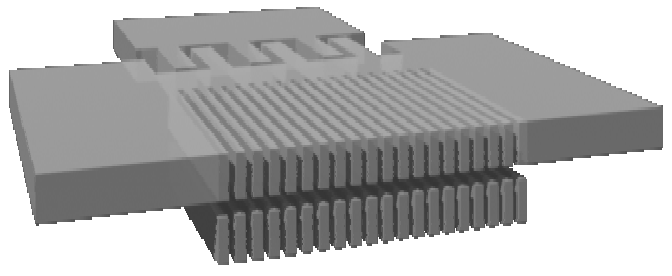

Figure 1. The device under consideration is a two layer grating in silicon with features much shorter than the wavelength of light. The two gratings are separated by a small air gap, and the upper grating can be moved horizontally. We can observe the motion by detecting reflected light from a normal incident beam.

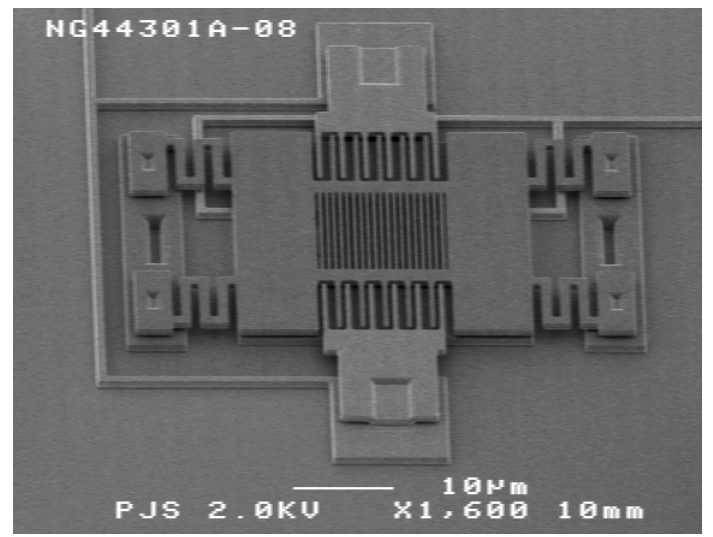

Figure 2. SEM micrograph showing a test device. The comb-drive fingers enable electrostatic actuation in the plane, and electrodes on the first level enable vertical actuation. The grating shown has a period of $720 \mathrm{~nm}$.

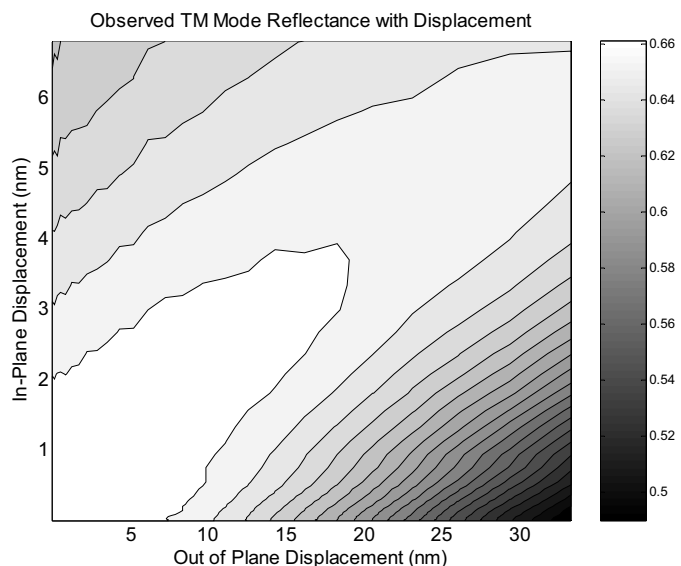

Figure 3. Measured reflectance from the surface of a device identical to the one shown in Figure 2. This is raw data that is acquired at a rate of $10 \mathrm{~Hz}$ while the lateral and vertical actuation voltages are swept, producing the displacements shown.

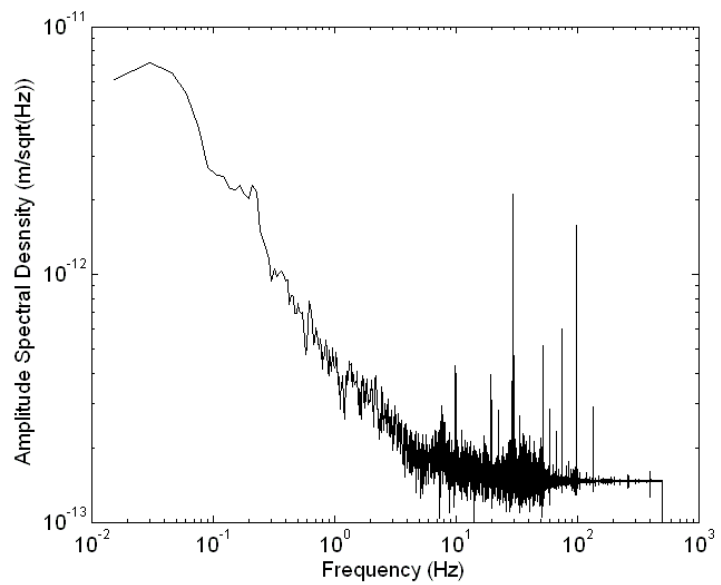

Figure 4. Spectral density plot showing the signal at $10 \mathrm{~Hz}$, and the noise floor of $140 \mathrm{fm} / \mathrm{Hz}^{1 / 2}$ at $10 \mathrm{~Hz}$. 